\title{
¿Qué textos leen en primer año los estudiantes de Psicología?
}

María Micaela Villalonga Penna'

Constanza Padilla'

\section{Resumen}

Qué se lee en las universidades es una de las temáticas que han abordado los estudios realizados desde los enfoques de las Alfabetizaciones Académicas y de la Literacidad crítica. En este sentido, nos preguntamos ¿qué textos leen en primer año los estudiantes de psicología? Teniendo en cuenta este interrogante, en este trabajo presentamos el análisis del material bibliográfico del primer año de la carrera de psicología de una universidad estatal. Esta investigación se realizó desde los enfoques cualitativo y cuantitativo de investigación. Analizamos un corpus de 320 textos que corresponden al material bibliográfico de las nueve materias del primer año de la mencionada carrera. Empleamos las categorías de análisis propuestas por Carlino (2005), Padilla (2013) y Padilla, Douglas y López (2007; 2011), entre otras, y tuvimos en cuenta categorías emergentes también. Los hallazgos dieron a conocer el uso de 'cuadernillos', que incluyen fotocopias del material bibliográfico que pueden o no contar con elementos que ayuden a contextuarlo como copias de la tapa y contratapa, página con datos de edición, índice y referencias completas. El material bibliográfico está constituido por textos académicos derivados de textos científicos, entre los que priman los capítulos de libros y las fichas de cátedra. Además, estos textos son predominantemente expositivos o argumentativos.

\section{Palabras Clave}

Lectura - Psicología - Primer Año - Material de enseñanza.

I- Universidad Nacional de Tucumán. Facultad de Psicología. Instituto de Investigaciones sobre el Lenguaje y la Cultura, CONICET., Argentina.

Contacto: micavillalonga@yahoo.com.ar 


\section{What texts do first-year Psychology students read?}

María Micaela Villalonga Penna'

Constanza Padilla'

\section{Abstract}

What is read in universities is one of the issues considered by studies carried out from the perspectives of Academic Literacies and the Critical Literacy. In this sense, we ask what texts first year Psychology students read. Considering such question, in this paper, we present the analysis of bibliographical material of the first year Psychology major of a public college. This is a quantitative and qualitative study. We have analyzed the 320 texts of the bibliographical material of the nine disciplines of the first year of Psychology major, not only using the categories proposed by Carlino (2005), Padilla (2013) and Padilla, Douglas and Lopez (2007, 2011), but also emergent categories. The results showed the use of booklets, which included photocopies of bibliographical material which may or may not have elements that could help readers contextualize it, such as copies of the front and back of the book, the page with edition data, content, and complete references. The bibliographical material consists of academic texts derived from scientific texts, especially book chapters and teaching slides. Alsok, those texts are predominantly expository or argumentative.

\section{Keywords}

Reading - Psychology - First Course - Teaching material.

I- Universidad Nacional de Tucumán. Facultad de Psicología. Instituto de Investigaciones sobre el Lenguaje y la Cultura, CONICET., Argentina.

Contact: micavillalonga@yahoo.com.ar 


\section{Introducción}

La lectura comprensiva en la universidad es una práctica social situada en el marco de comunidades discursivas. Desde el enfoque de las Alfabetizaciones académicas y de la Literacidad crítica se ha propuesto la existencia de diversos aspectos relacionados entre sí que hacen a la lectura académica, entre ellos, las características que poseen los textos disciplinares (BAZERMAN, 1988; BLOMMAERT et al., 2007; CARLINO, 2003a; 2003b; 2005; LEA, 2005; LILLIS; SCOTT, 2007; MARIN, 2006), los conocimientos acerca del código escrito y los géneros discursivos (BARTON; HAMILTON; IVANIC, 2000; BARTON; TUSTING, 2005; CASSANY, 2005). Como podemos apreciar, desde estos dos enfoques teóricos, a la hora de hablar de lectura en la universidad una de las cuestiones a tener en cuenta es qué se lee en las comunidades disciplinares. Por ello, en este trabajo nos interrogamos sobre qué características tienen los textos que incluye el material bibliográfico del primer año de la carrera de psicología de una universidad pública argentina.

Estudios regionales han reflexionado acerca de qué se lee en la universidad (BIGI; CHACÓN; GARCÍA, 2013; CARLINO, 2005; GIUDICE, 2014; GODOY; GIUDICE, 2014; PÉREZ ABRIL; RINCÓN BONILLA, 2013). En relación con esto, Carlino (2005) ha enfatizado que los alumnos universitarios en el área de ciencias sociales y humanidades suelen leer textos académicos derivados de textos científicos, definiendo a los textos académicos, como "aquellos que se utilizan para enseñar y aprender en la universidad" (CARLINO, 2005, p. 87). Los textos académicos derivados de textos científicos, a los que generalmente acceden, son los materiales de cátedra, los manuales, los libros y los capítulos de libros. Estos textos se caracterizan porque su fuente son trabajos científicos y, entre el autor y el lector, suele haber una asimetría de conocimientos que favorece al autor (CARLINO, 2003a; 2005).

Asimismo, señaló una práctica institucionalizada vinculada con el material bibliográfico: el uso de fotocopias de los textos académicos que se encuentran fuera de la obra completa. Los estudiantes no acceden a las obras originales y las fotocopias que leen no poseen los capítulos precedentes y subsiguientes; se encuentran desprovistas del índice, prólogo, introducciones, solapas que presentan a los autores, contratapas que comentan el texto, referencias bibliográficas, datos de la edición y título. Si bien estos recortes pueden deberse a cuestiones presupuestarias, también pueden atribuirse a la falta de cuidado de los docentes al preparar el material. Por ello, Carlino (2005) invita a reflexionar acerca de las dificultades que pueden presentarse a un lector para ubicarse en lo que lee, cuando el material bibliográfico proporcionado posee estas características.

En la misma dirección, Bigi, Chacón y García (2013) analizaron cuáles textos académicos se leen en la carrera de Educación de la Universidad de Los Andes. Para ello, aplicaron un cuestionario a 230 alumnos y a cuarenta docentes de la mencionada carrera durante el año 2012. El cuestionario administrado a los docentes incluía un ítem sobre los diferentes tipos de textos o géneros académicos. Las respuestas referían a la frecuencia de uso y contemplaban cinco opciones mutuamente excluyentes: siempre, casi siempre, algunas veces, pocas veces, nunca.

Hallaron que para la categoría generalmente, el 67\% de los estudiantes declara leer capítulos de libros fotocopiados; para la categoría, algunas veces, el 57,8\%, señala leer guías de textos elaboradas por los profesores. Para la categoría casi nunca, el 68,3\%, lee artículos científicos, el 63\%, artículos de opinión, el 61,7\%, informes de investigación y el 60,9\%, libros completos. Por su parte, los docentes indicaron, que mayoritariamente, se leen capítulos de libros y las páginas web (75\%); en menor medida, artículos científicos (55\%), artículos de opinión (50\%), diccionarios especializados (42,7\%) y libros $(42,7 \%)$; y pocas veces, ponencias (75\%), informes de investigación (52,5\%), notas de enciclopedias (50\%), guías de estudio (50\%), artículos de 
opinión (50\%), diccionarios especializados (45\%), y ensayos y libros (42,5\%). Con base en estos hallazgos, señalaron la escasa variedad de textos académicos que los docentes dan a sus alumnos para que lean, y reflexionaron acerca de las posibilidades de ingreso de los alumnos a una comunidad académica que forme lectores críticos.

En un estudio realizado por Pérez Abril y Rincón Bonilla (2013), en diversas universidades colombianas, indagaron qué leen los estudiantes de grado. A partir de una encuesta realizada a estudiantes, hallaron que el 84\% indica leer mayoritariamente apuntes de clase propios, el 80\% materiales elaborados por los profesores, el 77\% páginas webs y blogs, el 72\% capítulos de libros, el 60\% libros de consulta, el 41\% artículos científicos, el 37\% informes de investigación, el 33\% apuntes de clase tomados por otro.

Por otra parte, Padilla, Douglas y López (2007; 2011) han indicado que en medios académicos suelen circular textos expositivos y argumentativos. A este respecto, cabe retomar los aportes de Arnoux, Di Stefano y Pereira (2002) quienes entienden a la exposición y a la argumentación como dos polos de un continuum. Los géneros expositivo $\mathrm{y}$ argumentativo desarrollan un razonamiento sobre un tema, la solución de un problema o la fundamentación de una opinión. En este punto, proponen que los discursos razonados considerados individualmente pueden tender al polo expositivo-explicativo o al argumentativo. Además, los géneros discursivos se reconocen por su relación con determinadas prácticas sociales, su carácter oral o escrito, su formato o su paratexto, y por el predominio que en cada uno de ellos tiene una $\mathrm{u}$ otra secuencia.

Las mencionadas autoras han caracterizado a los polos expositivo-explicativo y argumentativo. En el caso del primero, se centra en un saber construido en otro lado y socialmente legitimado, buscando informar y tendiendo a borrar las huellas subjetivas para marcar una distancia y objetividad. Asimismo, en los textos expositivos se apela a citas de autoridad. En el caso del segundo, se buscar persuadir y el sujeto se manifiesta confrontando o no su opinión con la de otros a fin de construir nuevos conceptos o posturas; por ello en este discurso aparecen múltiples voces.

Los desarrollos de Padilla, Douglas y López $(2007$; 2011) sobre la existencia de textos predominantemente expositivos o argumentativos, se sitúan en la misma línea que los de Arnoux, Di Stefano y Pereira (2002). Estas autoras hablan de textos de predominio expositivo y de textos de predominio argumentativo y han señalado que la existencia de textos netamente expositivos o argumentativos suele ser poco frecuente. Además, han propuesto una serie de niveles para su análisis, que pueden ser útiles para distinguir si se trata de textos predominantemente expositivos o argumentativos.

En relación con el discurso expositivo, el nivel pragmático de este tipo discursivo, está referido a la intencionalidad (que apunta a hacer comprender un tema) y el contexto de producción (dado por los ámbitos de divulgación del conocimiento científico); el nivel global, a la estructura o modo de organización del discurso expositivo (descripción, seriación, organización causal, problema/solución, comparación); y el nivel local, a las estrategias discursivas -formas de impersonalidad (uso del se impersonal, del se pasivo y de la voz pasiva), omisión de las formas de modalización, uso de tiempos verbales del indicativo-. Sobre el discurso argumentativo, el nivel pragmático implica también, la intencionalidad y el contexto de producción, es decir, la forma en que se despliega la intención argumentativa para incidir en la forma de pensar y/o actuar del destinatario (estrategia justificativa, estrategia polémica, estrategia deliberativa); el nivel global, las categorías estructurales (tesis y conclusión, premisas y argumentos -mediante ejemplos, analogías, autoridad, causales-) y las estructuras alternativas de la argumentación para las diferentes estrategias argumentativas, y el nivel local del discurso argumentativo, las modalidades discursivas (modalidades de enunciación asertiva e interrogativa; modalidades del enunciado lógicas o intelectuales, apreciativas, valorativas, 
desiderativas, de necesidad; enunciados polifónicos como negaciones polémicas, estructuras adversativas y estructuras concesivas) (PADILLA; DOUGLAS; LÓPEZ, 2007; 2011).

Otro aporte significativo es el que realizan Kaufman y Rodríguez (1993), quienes han propuesto clasificar los textos según su función predominante y trama. En este caso, resulta de interés retomar el tipo de trama textual, ya que en algunos puntos presenta similitudes con lo señalado por Arnoux, Di Stefano y Pereira (2002) y por Padilla, Douglas y López $(2007,2011)$ y, en otros, apreciamos aspectos no explicitados por las antes mencionadas autoras. Hay cuatro tipo de tramas: la trama descriptiva -presenta caracterizaciones de personas, objetos, escenarios o procesos a través de sus rasgos o características distintivas-; la trama argumentativa -con base en un tema o una hipótesis, se organiza una serie de explicaciones, ejemplificaciones y confrontaciones de ideas, para llegar a una conclusión-; la trama narrativa -presenta hechos organizados en un eje temporal, personajes que realizan acciones y relaciones de tipo causaefecto-, y la trama dialogal (o conversacional) -se basa en un intercambio lingüístico entre dos o más participantes de una situación comunicativa- (KAUFMAN, 1994; KAUFMAN; RODRÍGUEZ, 1993).

Finalmente, retomamos las reflexiones de Carlino $(2005 ; 2010)$ y de Araujo y Gomes Bezerra (2013) quienes han propuesto que los docentes debieran considerar enseñar a los estudiantes a leer los textos académicos disciplinares ya que esto permitiría a los nóveles lectores poder incluirse en la comunidad lectora académica y disciplinar. Desde esta perspectiva, se ha criticado aquellas prácticas institucionales que consisten en relegar la enseñanza de la lectura académica a instancias de carácter remedial que se centran en la transmisión de destrezas lectoras de manera descontextualizada -es decir, desarticuladas de las asignaturas y disciplinas que se enseñan en los centros académicos- y que buscan paliar lo que los estudiantes no hubieran aprendido en el secundario en relación con la lectura (CARLINO, 2010). La importancia de esto puede verse, por ejemplo, en los desarrollos de estudios nacionales (ESTIENNE; CARLINO, 2004; FERNÁNDEZ; CARLINO, 2010; PADILLA; DOUGLAS; LÓPEZ, 2007; 2011), que han señalado que en carreras universitarias del área de las ciencias sociales suelen leerse múltiples fuentes textuales académicas que pueden ser de tipo argumentativo que se leen según las posturas de cátedras, y algunos docentes no logran visualizar esto con claridad o esperan que los estudiantes hayan aprendido en la escolaridad secundaria a leer teniendo en cuenta estos aspectos.

En relación con lo anterior, no podemos pasar por alto los desarrollos teóricos que han indicado que una verdadera inclusión en el sistema de educación universitaria no implica sólo acceder a la misma, sino tener en cuenta cuáles son los resultados en términos de permanencia y egreso del sistema y qué condiciones facilitan u obstaculizan esto (CHIROLEU, 2009a; 2009b). En este sentido, en América Latina así como las tasas de ingreso al Sistema Educativo Superior han aumentado, se aprecian también altas tasas de fracaso académico y abandono, que terminan perjudicando a amplias franjas sociales desfavorecidas en la distribución del capital económico y cultural (CHIROLEU, 2009a; EZCURRA, 2011). Según Ezcurra (2011) las brechas de graduación universitaria son también brechas de clase y esto la ha llevado a plantear que en el Sistema Educativo Superior se ha generado una inclusión excluyente. Teniendo en cuenta desarrollos de nuestro país (CARLINO, 2003; 2005; ESTIENNE; CARLINO, 2004) que señalan la relevancia de los procesos lectores como puerta de entrada a la cultura académica, podemos considerar que aquellos estudiantes que se encuentren menos familiarizados con las prácticas alfabetizadoras propias de las comunidades académicas, pueden llegar a correr el riesgo de no poder incluirse a menos que los docentes expliciten qué es lo que esperan de los estudiantes como lectores y los ayuden a construir aprendizajes en este sentido.

Teniendo en cuenta estos aportes, nos proponemos describir el material bibliográfico 
que se utiliza en nueve asignaturas del primer año de la carrera de psicología de una universidad pública argentina ${ }^{1}$.

\section{Metodología}

En este trabajo combinamos los enfoques cuantitativo y cualitativo de investigación (HERNÁNDEZ SAMPIERI; FERNÁNDEZ COLLADO; BAPTISTA LUCIO, 2006). Analizamos los textos que corresponden al material bibliográfico de las nueve materias del primer año de la citada carrera. Tres de estas materias son anuales (Introducción a la psicología, Psicofisiología y Psicología del curso vital I); cuatro son cuatrimestrales (Problemas sociológicos en psicología, Temas de antropología cultural en psicología, Temas de filosofía y lógica en psicología, Historia de la psicología) y dos son entre bi y trimestrales (Integración universitaria y seminario-taller: producción de textos cientificos). El corpus estuvo compuesto por 320 textos. En la tabla que sigue sintetizamos la composición del mismo.

Quadro 1- Estadísticos descriptivos para la cantidad de textos analizados por materia

\begin{tabular}{ccc:c}
\hline Materia & F & P \\
\hline Introducción a la psicología & $\vdots$ & 41 & 12,8 \\
\hline Psicofisiología & $\vdots$ & 38 & 11,9 \\
\hline Psicología del curso vital I & $\vdots$ & 96 & 30,0 \\
\hline Problemas sociológicos en psicología & $\vdots$ & 30 & 9,4 \\
\hline Temas de antropología cultural en psicología & 38 & 11,9 \\
\hline Historia de la psicología y del psicoanálisis & $\vdots$ & 24 & 7,5 \\
\hline Temas de filosofía y lógica para psicología & $\vdots$ & 27 & 8,4 \\
\hline Seminario de producción de textos científicos & 12 & 3,8 \\
\hline Integración universitaria & $\vdots$ & 14 & 4 \\
\hline Total & 320 & 100,0 \\
\hline
\end{tabular}

Fuente: elaboración propia.

F: frecuencia

P: porcentaje

1- Cabe señalar que el acceso a esta carrera no posee restricciones y la primera materia que los estudiantes cursan es la Integración univeristaria -que es bimestral y obligatoria-no eliminatoria-. Además, la facultad de psicología recibe una población estudiantil muy heterogénea procedente tanto de la provincia en la que está ubicada como de provincias cercanas.
Analizamos los textos teniendo en cuenta categorias ya propuestas por autores y categorías emergentes. Asimismo, de forma cuantitativa, apelamos a herramientas de estadística descriptiva (frecuencias y porcentajes) y nos apoyamos en el SPSS (Statistical Package for Social Sciences).

\section{Organización del material de lectura}

Contempla forma de presentación del material bibliográfico y cómo ha sido estructurado. Esta categoría a su vez comprende sub-categorías:

1) Cuadernillos: compilación de fotocopias de textos académicos derivados de textos científicos y de textos científicos (CARLINO, 2005). Para esta sub-categoría, las opciones de respuesta fueron: usan cuadernillos, no usan cuadernillos, no usan cuadernillos pero circulan cuadernillos no oficiales. Contabilizamos las frecuencias de ausencias, presencias y de no correspondencias.

Además, tuvimos en cuenta una serie de sub-categorias emergentes referidas la inclusión de:

2) Programas de las materias: incluyen los contenidos temáticos organizados en ejes o unidades, la bibliografía correspondiente obligatoria y/o ampliatoria-, guías de trabajo, fechas relevantes del cursado.

3) Carátula con el nombre de la materia: página que contiene el nombre de la asignatura y que se ubica en primer lugar en el cuadernillo.

4) Separadores bibliográficos: páginas que se ubican para diferenciar la bibliografía de las unidades temáticas.

5) Características de los separadores: hace referencia al tipo de información que incluyen los separadores bibliográficos.

6) Completud del material: inclusión de clases de textos que pueden estar completos o incompletos -o truncados-.

Para programas de materias las opciones de respuesta fueron incluido en el cuadernillo $y$ no incluido en el cuadernillo; para carátula con el nombre de la materia, incluye y no incluye; para 
separadores bibliográficos por unidades temáticas, incluye y no incluye; para características de los separadores, incluye sólo la unidad temática, incluye la unidad temática, la bibliografía $y / 0$ los contenidos, no corresponde -en aquellos casos en los que el material bibliográfico no incluía separadores-. Para completud del material las opciones de respuesta fueron completo e incompleto. Tomamos la frecuencia de presentación de cada una de las respuestas.

\section{Elementos para contextuar lo que se lee}

Implica una serie de sub-categorías: 1) tapas; 2) contratapas que comenten la obra; 3) solapas que presenten a los autores; 4) página con datos de edición; 4) índice; 5) introducción y/o prólogo; 6) bibliografía (CARLINO, 2005). Los textos se clasificaron teniendo en cuenta la presencia o la ausencia de estos elementos según correspondiera. Además, se incluyó la opción no corresponde para dar cuenta de aquellas publicaciones que en su versión original no incluían alguno de los elementos. Se tomó la frecuencia de presentación de cada opción de respuesta (presencia-ausencia-no corresponde) para cada elemento o sub-categoría y el porcentaje. Se agregó como categoría emergente la de referencias bibliográficas. Las opciones de respuesta fueron: referencia completa, referencia incompleta y no posee referencia. Contabilizamos la frecuencia de presentación de cada clase de respuesta y calculamos el porcentaje.

\section{Clases de textos}

Clases de textos incluidos en el material bibliográfico de las materias. Esta categoría comprende las siguientes sub-categorías.

1) textos académicos derivados de textos científicos: (libros, capítulos de libros, materiales de cátedra, manuales) (CARLINO, 2005; PADILLA, 2013).

2) textos científicos (artículos de revistas de investigación, tesis, ponencias presentadas en congresos, informes, proyectos de investigación) (CARLINO, 2005; PADILLA, 2013).
Además, agregamos como sub-categorías emergentes:

3) artículos de divulgación;

4) transcripciones de clases o conferencias;

5) entrevistas: transcripciones de entrevistas a personas reconocidas en un ámbito disciplinar.

Calculamos la frecuencia de presentación y el porcentaje de cada uno de estos textos en el material bibliográfico.

\section{Tipo discursivo predominante}

Esta categoria comprende subcategorías: 1) predominantemente expositivo; 2) predominantemente argumentativo; 3) con predominio de argumentación cotidiana, (PADILLA; DOUGLAS; LÓPEZ, 2007; 2011); 4) conversacionales (KAUFMAN; RODRÍGUEZ, 1993).

Se clasificaron los textos según el predominio discursivo y se contabilizó la frecuencia de los mismos y el porcentaje.

De forma cualitativa se analizaron los tipos discursivos predominantes incluyendo ejemplos extraídos de los textos. Para ellos se consideraron las siguientes categorías propuestas por Padilla, Douglas y López (2007; 2011):

- Nivel pragmático del discurso expositivo: intencionalidad comunicativa del enunciador en relación con el contexto de producción.

- Nivel global del discurso expositivo: estructura o modo de organización del discurso expositivo (descripción, seriación, organización causal, problema/solución, comparación).

- Nivel local del discurso expositivo (estrategias discursivas): formas de impersonalidad (uso del se impersonal, del se pasivo y de la voz pasiva), omisión de las formas de modalización, uso de tiempos verbales del indicativo.

- Nivel pragmático del discurso argumentativo (intencionalidad y contexto). Forma en que se despliega la intención argumentativa para incidir en la forma de pensar y/o actuar del destinatario (estrategia justificativa, estrategia polémica, estrategia deliberativa). 
- Nivel global del discurso argumentativo: categorías estructurales (tesis y conclusión, premisas, argumentos -mediante ejemplos, analogías, de autoridad, causales-), estructuras alternativas de la argumentación para las diferentes estrategias argumentativas.

- Nivel local del discurso argumentativo: modalidades discursivas (modalidades de enunciación asertiva e interrogativa; modalidades del enunciado lógicas o intelectuales, apreciativas, valorativas, desiderativas, de necesidad; enunciados polifónicos como negaciones polémicas, estructuras adversativas y estructuras concesivas).

Además, se tuvieron en cuenta categorías ya citadas: clases de textos con sus sub-categorías correspondientes (textos académicos derivados de textos científicos, textos científicos, artículos de divulgación, transcripciones de clases o conferencias, entrevistas), tipo de fuente (primaria, secundaria), completud del material y elementos para contextuar el material de lectura (tapa, contratapa, página con datos de edición, índice, introducción).

\section{Resultados y discusiones}

Para describir el material bibliográfico, hemos tenido en cuenta la organización del mismo, si contenían elementos que permitieran contextuar el material de lectura, el tipo de textos incluidos, el tipo discursivo predominante en los textos.

\section{¿Cómo se encuentra organizado el material bibliográfico?}

En primerlugar, en cuanto ala organización del material bibliográfico, observamos que en todas las asignaturas relevadas (nueve materias), el mismo se encuentra contenido en cuadernillos que contienen fotocopias del mismo. Sólo en un caso, se ha detectado la circulación de cuadernillos no oficiales o no preparados por la cátedra correspondiente, y que es utilizado por los estudiantes.
En segundo lugar, hallamos que casi todas las materias (ocho de las nueve materias) han incluido en los cuadernillos carátulas que indican el nombre de la materia, el año de cursado y la facultad y universidad y, sólo una de ellas (una de las nueve materias) no lo ha hecho. La mayor parte de las materias (seis de las nueve materias) han incluido separadores entre el material bibliográfico de las diferentes unidades temáticas $\mathrm{y}$, las menos (tres de las nueve materias), no lo han hecho. Estos separadores adoptan dos formas: por un lado, como sumarios bibliográficos de cada unidad temática del programa, con el título de la unidad y su numeración (dos de las nueve materias); y por el otro, como indicadores con el título y numeración de la unidad solamente (cuatro de las nueve materias).

En tercer lugar, en general (seis de las nueve materias), los cuadernillos incluyen el programa de las materias. Sin embargo, en aquellos casos en que el programa no ha sido incluido (tres de las nueve materias), el mismo se encuentra a disposición de los alumnos. La organización de los programas, en general (seis de las nueve materias), posee los contenidos temáticos $\mathrm{y}$ la bibliografía correspondiente en el mismo apartado. Pero, en algunos casos (tres de las nueve materias), los contenidos temáticos y la bibliografía correspondiente se encuentran en apartados diferentes. En pocos casos (dos de las nueve materias), los programas de las asignaturas van acompañados de guías de trabajo sobre el material bibliográfico, y de un anexo con fechas y temáticas de las clases teóricas y fechas tentativas de los exámenes parciales (tres de las nueve materias).

En cuarto lugar, otro aspecto que tuvimos en cuenta fue si los textos que componen el material bibliográfico de todas las materias, se encontraban completos o incompletos. La mayor parte del material bibliográfico se encuentra completo $(83,4 \%) \mathrm{y}$, la menor parte $(16,6 \%)$ está incompleta o truncada.

En la misma dirección que Carlino (2005) podemos apreciar que los alumnos no acceden a las obras originales y trabajan con fotocopias contenidas en cuadernillos en 
las diferentes asignaturas. El hecho de que en estos cuadernillos se haya incluido, en general, una carátula y separadores temáticos puede facilitar a los alumnos identificar el material bibliográfico. Sin embargo, en el caso del material bibliográfico incompleto, nos preguntamos cómo puede llegar a incidir en las posibilidades de construir la coherencia textual de los nóveles lectores de textos académicos.

\section{¿Con qué elementos cuentan los estudiantes para contextuar lo que leen?}

Como se muestra en la Tabla 1, analizamos si el material bibliográfico de todas las materias tenía elementos que ayudaran al lector a contextuarlo. En general, la tapa, contratapa, solapas, página con datos de edición, indice, bibliografía se encuentran ausentes.

Tabla 1- Estadísticos descriptivos para los elementos que ayudan a contextuar los textos en el material bibliográfico en general.

\begin{tabular}{|c|c|c|c|}
\hline Elementos para contextuar lo que se lee & Categorías & $\mathrm{F}$ & $P$ \\
\hline \multirow{4}{*}{ Tapa del libro o revista } & Presente & 98 & 30,6 \\
\hline & Ausente & 162 & 50,6 \\
\hline & No corresponde & 60 & 18,8 \\
\hline & Total & 320 & 100,0 \\
\hline \multirow{4}{*}{ Contratapa del libro o revista } & Presente & 16 & 5,0 \\
\hline & Ausente & 243 & 75,9 \\
\hline & No corresponde & 61 & 19,1 \\
\hline & Total & 320 & 100,0 \\
\hline \multirow{3}{*}{ Solapas del libro o revista } & Ausente & 170 & 53,1 \\
\hline & No corresponde & 150 & 46,9 \\
\hline & Total & 320 & 100,0 \\
\hline \multirow{4}{*}{ Página con datos de edición } & Presente & 54 & 16,9 \\
\hline & Ausente & 205 & 64,1 \\
\hline & No corresponde & 61 & 19,1 \\
\hline & Total & 320 & 100,0 \\
\hline \multirow{4}{*}{ Bibliografía } & Presente & 98 & 30,6 \\
\hline & Ausente & 197 & 61,6 \\
\hline & No corresponde & 25 & 7,8 \\
\hline & Total & 320 & 100,0 \\
\hline \multirow{4}{*}{ Índice del libro o revista } & Presente & 18 & 5,6 \\
\hline & Ausente & 244 & 76,3 \\
\hline & No corresponde & 58 & 18,1 \\
\hline & Total & 320 & 100,0 \\
\hline
\end{tabular}

Fuente: elaboración propia.

F: frecuencia

P: porcentaje 
También tuvimos en cuenta si las referencias del texto estaban completas o no. A este respecto, hallamos que el 57,5\% de los textos tenían las referencias incompletas, el $31,3 \%$, las tenían completas y el 11,3\%, no tenían referencias. En este último caso suelen omitirse datos esenciales como el año de publicación, el título de la obra, el lugar de edición y la editorial, el título del capítulo y las páginas seleccionadas.

En la misma dirección que los hallazgos de Carlino (2005), los cuadernillos suelen no incluir fotocopia de la tapa, la contratapa con comentarios del texto, las solapas con información de los autores, la página con datos de edición, el índice de la obra que permita conocer el contenido global, la bibliografía que posibilite conocer las fuentes en las que se basa el material. A esto puede agregarse que las referencias bibliográficas suelen aparecer de forma incompleta. Esto podría incidir negativamente en las posibilidades de los nóveles lectores para contextuar la obra y para motivarse con la lectura de obras completas o valorar la relevancia de los aportes que retoman los autores para sustentar sus planteos y profundizar en los mismos. En este sentido, creemos que al preparar el material de lectura para los estudiantes los docentes deberían incluir este tipo de elementos y enseñar en las aulas universitarias cuál es el valor y la funcionalidad de los mismos en el marco de una disciplina y de un programa de estudios, a fin de facilitar que los estudiantes construyan no sólo del sentido de lo que leen en el marco de una materia, sino también de una disciplina.

\section{¿Qué clases de textos leen los alumnos?}

Tal como se muestra en la Tabla 2, abordamos el tipo de textos académicos incluidos por materia. Como se ve en esta tabla, predominan los textos académicos derivados de textos científicos, y entre éstos, los capítulos de libros y las fichas de cátedra. En este sentido, apreciamos que los alumnos leen, en mayor medida, capítulos de libros (64,9\%); en menor cantidad, fichas de cátedra (14,7\%), materiales de cátedra -como definiciones, imágenes y clasificaciones- (11\%), introducciones de libros (13\%), transcripciones de clases o conferencias (15\%); y casi nada, trabajos monográficos $(0,3 \%)$, ensayos $(0,7 \%)$ y artículos de divulgación (0,7\%).

Tabla 2- Estadísticos descriptivos para la clase de textos en el corpus general

\begin{tabular}{|c|c|c|}
\hline Clases de texto & $\mathrm{F}$ & $P$ \\
\hline Capítulo de libro & 204 & 63,8 \\
\hline Ficha de cátedra & 47 & 14,7 \\
\hline Artículo de divulgación & 2 & ,6 \\
\hline Artículo científico & 9 & 2,8 \\
\hline Transcripción de conferencia o clase & 15 & 4,7 \\
\hline Trabajo científico en actas de encuentro & 12 & 3,8 \\
\hline Introducción de libro & 14 & 4,4 \\
\hline $\begin{array}{l}\text { Material de cátedra (definiciones, } \\
\text { clasificaciones, imágenes) }\end{array}$ & 11 & 3,4 \\
\hline Ensayo & 2 & 6 \\
\hline Trabajo monográfico & 1 & ,3 \\
\hline Libro & 2 & ,6 \\
\hline Entrevista & 1 & ,3 \\
\hline Total & 320 & 100,0 \\
\hline
\end{tabular}

Fuente: elaboración propia.

F: frecuencia

P: porcentaje

Además, buscamos analizar las clases de textos utilizados en las diferentes materias, lo cual puede verse en la Tabla 3.

En general, en los cuadernillos de las diferentes asignaturas predominan los textos académicos derivados de textos científicos -como los capítulos de libros y las fichas de cátedra-. Todo esto se sitúa en la misma línea de que lo descripto por Carlino (2005). Aunque en el estudio citado de Bigi, Chacón y García (2013) se han indagado a docentes y estudiantes de forma directa para conocer qué se lee en la universidad, consideramos que sus aportes resultan importantes en esta discusión porque, en consonancia con estos autores, en nuestro corpus priman los capítulos 
Tabla 3- Estadísticos descriptivos para las clases de textos por materia

\begin{tabular}{|c|c|c|c|}
\hline Materia & Clase de texto & $\mathrm{F}$ & $\mathrm{P}$ \\
\hline \multirow{5}{*}{ Introducción a la psicología } & Capítulo de libro & 35 & 85,4 \\
\hline & Ficha de cátedra & 1 & 2,4 \\
\hline & Transcripción de conferencia o clase & 4 & 9,8 \\
\hline & Introducción de libro & 1 & 2,4 \\
\hline & Total & 41 & 100,0 \\
\hline \multirow{4}{*}{ Psicofisiología } & Capítulo de libro & 24 & 63,2 \\
\hline & Ficha de cátedra & 5 & 13,2 \\
\hline & Material de cátedra (definiciones, clasificaciones, imágenes) & 9 & 23,7 \\
\hline & Total & 38 & 100,0 \\
\hline \multirow{8}{*}{ Psicología del curso vital I } & Capítulo de libro & 37 & 38,5 \\
\hline & Ficha de cátedra & 34 & 35,4 \\
\hline & Artículo de divulgación & 1 & 1,0 \\
\hline & Artículo científico & 3 & 3,1 \\
\hline & Transcripción de conferencia o clase & 11 & 11,5 \\
\hline & Trabajo científico en actas de encuentro & 6 & 6,3 \\
\hline & Introducción de libro & 4 & 4,2 \\
\hline & Total & 96 & 100,0 \\
\hline \multirow{4}{*}{ Problemas sociológicos en psicología } & Capítulo de libro & 24 & 80,0 \\
\hline & Introducción de libro & 5 & 16,7 \\
\hline & Ensayo & 1 & 3,3 \\
\hline & Total & 30 & 100,0 \\
\hline \multirow{5}{*}{ Temas de antropología cultural en psicología } & Capítulo de libro & 35 & 92,1 \\
\hline & Artículo científico & 1 & 2,6 \\
\hline & Trabajo científico en actas de encuentro & 1 & 2,6 \\
\hline & Introducción de libro & 1 & 2,6 \\
\hline & Total & 38 & 100,0 \\
\hline \multirow{8}{*}{ Historia de la psicología } & Capítulo de libro & 12 & 50,0 \\
\hline & Ficha de cátedra & 2 & 8,3 \\
\hline & Artículo científico & 3 & 12,5 \\
\hline & Trabajo científico en actas de encuentro & 3 & 12,5 \\
\hline & Introducción de libro & 1 & 4,2 \\
\hline & Libro & 2 & 8,3 \\
\hline & Entrevista & 1 & 4,2 \\
\hline & Total & 24 & 100,0 \\
\hline \multirow{5}{*}{ Temas de filosofía y lógica para psicología } & Capítulo de libro & 22 & 81,5 \\
\hline & Ficha de cátedra & 2 & 7,4 \\
\hline & Artículo científico & 1 & 3,7 \\
\hline & Introducción de libro & 2 & 7,4 \\
\hline & Total & 27 & 100,0 \\
\hline \multirow{5}{*}{ Seminario de producción de textos científicos } & Capítulo de libro & 8 & 66,7 \\
\hline & Trabajo científico en actas de encuentro & 1 & 8,3 \\
\hline & Material de cátedra (definiciones, clasificaciones, imágenes) & 2 & 16,7 \\
\hline & Trabajo monográfico & 1 & 8,3 \\
\hline & Total & 12 & 100,0 \\
\hline \multirow{7}{*}{ Integración universitaria } & Capítulo de libro & 7 & 50,0 \\
\hline & Ficha de cátedra & 3 & 21,4 \\
\hline & Artículo de divulgación & 1 & 7,1 \\
\hline & Artículo científico & 1 & 7,1 \\
\hline & Trabajo científico en actas de encuentro & 1 & 7,1 \\
\hline & Ensayo & 1 & 7,1 \\
\hline & Total & 14 & 100,0 \\
\hline
\end{tabular}

Fuente: elaboración propia. F: frecuencia P: porcentaje 
de libros. Sin embargo, a diferencia de lo hallado por estas autoras, las páginas web en nuestra investigación no constituyen parte significativa del material bibliográfico de las materias. Asimismo, si bien el estudio encarado por Pérez Abril y Rincón Bonilla (2013) presenta diferencias sustantivas a nivel metodológico, señalamos que a diferencia de lo hallado por estos autores en nuestro corpus priman los capítulos de libro y las fichas de cátedra. Creemos que tanto docentes como alumnos debieran ser conscientes de las diferencias entre textos científicos y textos académicos que derivan de textos científicos, ya que sus características discursivas difieren y su nivel de complejidad también y esto es algo que sería necesario que los docentes expliciten a los estudiantes.

\section{¿Qué tipo discursivo predomina en los textos que leen los estudiantes?}

En la Tabla 4 puede observarse que en el material bibliográfico en general priman los textos de predominio expositivo.

Tabla 4- Estadísticos descriptivos para el tipo discursivo en el corpus en general

\begin{tabular}{|c|c|c|}
\hline Tipo discursivo & $\mathrm{F}$ & $\mathrm{P}$ \\
\hline De predominio expositivo & 210 & 65,6 \\
\hline De predominio argumentativo & 99 & 30,9 \\
\hline De predominio narrativo & 1 & , 3 \\
\hline $\begin{array}{c}\text { Con predominio de argumentación } \\
\text { cotidiana }\end{array}$ & 9 & 2,8 \\
\hline Conversacional & 1 & ,3 \\
\hline Total & 320 & 100,0 \\
\hline
\end{tabular}

Fuente: elaboración propia.

F: frecuencia

P: porcentaje

Analizamos también el tipo discursivo predominante en el material bibliográfico por materias, lo cual se presenta en la Tabla 5.

Como podemos apreciar en casi todas las materias priman los textos predominantemente expositivos sobre los textos predominantemente argumentativos, excepto en Introducción a la psicología en la cual priman los textos de predominio argumentativo y en Temas de antropología cultural y en Psicología del curso vital I, en las cuales la cantidad de textos de ambos tipos discursivos resulta casi equivalente. Cabe señalar también que en una materia -Psicofisiología- los estudiantes leen exclusivamente textos de predominio expositivo y que en otras materias se leen textos narrativos, dialogales y con predominio de argumentación cotidiana. Además, en el Seminario de producción de textos científicos se trabaja sólo con un texto de predominio argumentativo, siendo que, en general, los textos científicos se caracterizan por ser predominantemente argumentativos.

Con base en las categorías analíticas de Padilla, Douglas y López (2007; 2011), se encontró que en materias como Integración universitaria, Psicofisiología, Psicología del curso vital I, Problemas sociológicos en psicología y Temas de antropología cultural en psicología abundan los textos de predominio expositivo, y en Introducción a la psicología, los de predominio argumentativo. Siguiendo a Padilla, Douglas y López (2011) también, apreciamos la presencia de textos en los que predomina la argumentación cotidiana en una materia Temas de antropología cultural en psicología-. Teniendo en cuenta los planteos de Kaufman y Rodríguez (1993) encontramos la presencia de textos conversacionales en Temas de filosofía $y$ Lógica para psicología.

Teniendo en cuenta el análisis cuantitativo del material bibliográfico expuesto en este subapartado, el predominio discursivo de los textos en las diferentes asignaturas, y la propuesta metodológica de este estudio, hemos analizado cualitativamente el tipo discursivo predominante en los textos, lo cual se presenta en los párrafos que siguen.

Un texto académico predominantemente expositivo, propio del campo de la psicología, es un capítulo de libro de Manuel De Vega $(1984)^{2}$ Introducción a la psicología cognitiva, en el cual

2- El citado texto se lee en la asignatura Introducción a la psicología, que es anual y de carácter obligatorio. 
Tabla 5- Estadísticos descriptivos para el tipo discursivo por materia

\begin{tabular}{|c|c|c|c|}
\hline Materia & Tipo discursivo & $\mathrm{F}$ & $P$ \\
\hline \multirow{3}{*}{ Introducción a la psicología } & De predominio expositivo & 20 & 48,8 \\
\hline & De predominio argumentativo & 21 & 51,2 \\
\hline & Total & 41 & 100,0 \\
\hline Psicofisiología & De predominio expositivo & 38 & 100,0 \\
\hline \multirow{3}{*}{ Psicología del curso vital I } & De predominio expositivo & 57 & 59,4 \\
\hline & De predominio argumentativo & 39 & 40,6 \\
\hline & Total & 96 & 100,0 \\
\hline \multirow{3}{*}{ Problemas sociológicos en psicología } & De predominio expositivo & 22 & 73,3 \\
\hline & De predominio argumentativo & 8 & 26,7 \\
\hline & Total & 30 & 100,0 \\
\hline \multirow{5}{*}{ Temas de antropología cultural en psicología } & De predominio expositivo & 15 & 39,5 \\
\hline & De predominio argumentativo & 13 & 34,2 \\
\hline & De predominio narrativo & 1 & 2,6 \\
\hline & Con predominio de argumentación cotidiana & 9 & 23,7 \\
\hline & Total & 38 & 100,0 \\
\hline \multirow{3}{*}{ Historia de la psicología } & De predominio expositivo & 17 & 70,8 \\
\hline & De predominio argumentativo & 7 & 29,2 \\
\hline & Total & 24 & 100,0 \\
\hline \multirow{4}{*}{ Temas de filosofía y lógica para psicología } & De predominio expositivo & 17 & 63,0 \\
\hline & De predominio argumentativo & 9 & 33,3 \\
\hline & Dialogal & 1 & 3,7 \\
\hline & Total & 27 & 100,0 \\
\hline \multirow{3}{*}{ Seminario de producción de textos científicos } & De predominio expositivo & 11 & 91,7 \\
\hline & De predominio argumentativo & 1 & 8,3 \\
\hline & Total & 12 & 100,0 \\
\hline \multirow{3}{*}{ Integración universitaria } & De predominio expositivo & 13 & 92,9 \\
\hline & De predominio argumentativo & 1 & 7,1 \\
\hline & Total & 14 & 100,0 \\
\hline
\end{tabular}

Fuente: elaboración propia.

F: frecuencia

P: porcentaje 
se desarrollan conceptos relevantes inherentes a la psicología cognitiva en tanto disciplina. Se trata de un texto académico derivado de textos científicos; se encuentra completo $\mathrm{y}$, si bien no viene acompañado de copia de elementos que ayuden a contextuarlo, posee referencias completas. Este texto, desde la perspectiva del propio autor, constituye un material o manual introductorio para aquellos que deseen conocer de qué se trata la psicología cognitiva. En este sentido, el capítulo seleccionado como material bibliográfico para los alumnos de primer año de la carrera de psicología es, como ya se indicó, una introducción en la que se explicitan algunos conceptos capitales de la psicología del conocimiento, se contextúan históricamente estos desarrollos, se marcan puntos de unión y de divergencia con otras disciplinas y teorías.

Además, mientras el autor desarrolla estas cuestiones, en algunos casos, va indicando en qué capítulos del libro se amplía lo que expone. Otra característica a señalar es que al finalizar el capítulo, el autor ofrece un panorama del texto en el que explicita algunos conceptos que ya venía utilizando a lo largo del capítulo, como los de estructuras, procesos y representaciones, y la organización del manual para facilitar el uso del mismo.

En este texto, los paratextos o imágenes incluidos complementan y amplían las explicaciones de algunos procesos experimentales, como los estadios de la simulación graficados en un diagrama de flujo. Otro aspecto a tener en cuenta, es el referido a cómo el autor resalta nombres propios a fin de llamar la atención del lector que se introduce en este campo disciplinar. Para ello, los nombres propios de autores se encuentran en letras mayúsculas de molde o imprenta, y las referencias a terminología disciplinar relevante (paradigma cognitivo, hardware, software, modelos, representación) en itálicas.

En lo referido a las estrategias discursivas, el autor emplea formas de impersonalidad, como el se impersonal: "Se creía que de este modo se podian aislar mejor las leyes de la memoria, pues la experiencia previa quedaba controlada" (DE
VEGA, 1984, p. 51). En algunos casos utiliza el nosotros de autor como una forma de invitar al lector a involucrarse en lo que desarrolla:

Vamos a describir dos tipos de paradigmas experimentales utilizados con profusión por la psicología cognitiva: los estudios cronométricos y los paradigmas de aprendizaje verbal. (DE VEGA, 1984, p. 45).

Se encuentran escasas referencias de modalización. Predomina el uso de tiempos verbales del indicativo: "Algunos psicólogos consideran que la transición del conductismo al cognitivismo se puede interpretar en el marco de la concepción kuhniana” (DE VEGA, 1984, p. 26). Además, incluye marcadores para organizar y enumerar información: "Los paradigmas básicos de aprendizaje verbal pueden describirse según tres criterios: a) según la estructura del material de aprendizaje; b) según el contenido; y c) según el procedimiento de la prueba de memoria” (p. 50).

Usa citas de autoridad para ratificar o sustentar lo que expone, como la de Kosslyn, sobre una de las características del sistema cognitivo que viene desarrollando De Vega, la interactividad, y las complicaciones que ésta genera para el investigador que desea estudiar aspectos de la arquitectura cognitiva:

\begin{abstract}
El psicólogo cognitivo a menudo tratará de estudiar algún aspecto particular de la cognición, tal como la naturaleza de las representaciones internas. Desgraciadamente cada aspecto está engranado en un sistema total de mecanismo. Para examinar cualquier componente, necesariamente incluiremos la codificación, la representación, la computación y los procesos de respuesta, todos los cuales se reflejan en nuestros datos (KOSSLYN, 1978, apud DE VEGA, 1984, p. 24).
\end{abstract}

Los ejemplos incluidos, en general, resultan explicativos y amplían información acerca de diversas cuestiones, como el sesgo derivado de la analogía mente-ordenador en las investigaciones: 
"Se ha dedicado un esfuerzo excesivo en los años 60 al estudio de las estructuras de la memoria y de la atención, mimetizando las características del hardware de los ordenadores" (p. 33).

A nivel pragmático, este texto posee referencias completas que pueden ayudar al lector a contextuarlo y su intención expositiva es presentada por el autor y puede apreciarse al leerlo, lo cual resulta concordante con lo señalado por Padilla, Douglas y López (2007) y por Arnoux, Di Stefano y Pereira (2002). A nivel global, es un texto predominantemente descriptivo (PADILLA; DOUGLAS; LÓPEZ, 2007) y, a nivel local, emplea las estrategias discursivas propias de la exposición.

Como ya se ha indicado, la selección bibliográfica incluye también textos de predominio argumentativo como el de Alfred Schutz (1974) "El forastero. Ensayo de psicología social". Se trata de un texto académico que se encuentra completo así como sus referencias, pero no se encuentra acompañado de otros elementos que faciliten contextuarlo.

En relación con las categorías estructurales, pueden reconocerse las categorías estructurales argumentativas y un modo de organización justificativa (PADILLA; DOUGLAS; LÓPEZ, 2011). La tesis de la que parte, consiste en que aquel que desea integrarse en un grupo social debe aprender a interpretar el esquema cultural del nuevo grupo y la conclusión a la que arriba confirma dicha tesis. El autor plantea premisas como:

El sistema de conocimiento... adquiere para los miembros del endogrupo la apariencia de una coherencia, claridad y congruencia suficientes como para ofrecer a cualquiera una probabilidad razonable de comprender y de ser comprendido. (SCHUTZ, 1974, p. 98).

Estas premisas se ven sustentadas por dos clases de argumentos. Por una parte, argumentos mediante analogías: "Este pensar habitual...corresponde a la idea de Max Scheler

3- El citado texto se lee en Problemas sociológicos en psicología, materia cuatrimestral de carácter obligatorio. de la concepción relativamente natural del mundo..." (SCHUTZ, 1974, p. 99). Por la otra, argumentos causales:

El pensar habitual puede ser mantenido mientras confirmen su validez ciertos supuestos básicos: 1) que la vida...seguirá siendo la misma que hasta ahora...; 2) que podemos confiar en el conocimiento recibido de nuestros padres, maestros...; 3) que en el curso ordinario de las cosas...basta saber algo de su tipo o estilo general; 4) que ni los sistemas de recetas...ni los supuestos básicos..., son asunto privado nuestro, sino que son aceptados y aplicados por nuestros semejantes. (SCHUTZ, 1974, p. 99).

Finalmente, son menos los casos en los que el autor apela a argumentos mediante ejemplos.

Las estrategias discursivas se caracterizan por la presencia de modalidades de enunciación asertivas:

Todo miembro nacido o educado dentro del grupo acepta el esquema estandarizado, ya elaborado de la pauta cultural recibida de sus antepasados, maestros y autoridades, como guía indiscutida e indiscutible en todas las situaciones que se dan normalmente dentro del mundo social. (SCHUTZ, 1974, p. 98).

También, por modalidades de enunciado lógicas como "Lamentablemente la dudosa lealtad del forastero suele ser algo más que un prejuicio por parte del grupo abordado" (p. 106), y valorativas, por ejemplo:

Como conveniente punto de partida investigaremos como se presenta la pauta cultural de la vida del grupo ante el sentido común de un hombre que vive su vida cotidiana dentro del grupo, entre sus semejantes. (SCHUTZ, 1974, p. 96).

Asimismo, se aprecian enunciados polifónicos adversativos como las negaciones polémicas: 
No queremos hacer excesivo hincapié en esta imagen, que ha tenido como propósito principal poner en claro que el conocimiento del hombre que actúa y piensa dentro del mundo de su vida cotidiana no es homogéneo... (SCHUTZ, 1974, p. 97).

y las estructuras adversativas:

La actitud crítica del forastero no basta para explicar su objetividad...Pero esta actitud se origina menos en su propensión a juzgar al nuevo grupo según las normas que trae de su grupo de origen...Sin embargo, la razón más profunda de su objetividad reside en su propia amarga experiencia de los límites del pensar habitual. (SCHUTZ, 1974, p. 106).

El autor apela asimismo al uso de la primera persona del plural o nosotros de autor, como una forma de acercarse al lector e involucrarlo, especialmente cuando propone argumentos a favor o en contra de su tesis.

El texto argumentativo, posee las categorías estructurales (tesis y conclusión, premisas y argumentos -mediante ejemplos, analogías, autoridad, causales-) propuestas por Padilla, Douglas y López (2011), y las estrategias discursivas empleadas a nivel local son propias de la argumentación académica. Asimismo, a nivel pragmático, y por la forma en que se han organizado las categorías estructurales, parece tener una estructura predominantemente justificativa (PADILLA; DOUGLAS; LÓPEZ, 2011). Un aspecto a considerar, es que en el citado ensayo se aborda una problemática contrastando diferentes fuentes en función de ejes temáticos e intentando convencer al lector con diversos argumentos. Esto resulta concordante con lo indicado por Arnoux, Di Stefano y Pereira (2002) y por Padilla, Douglas y López $(2007,2011)$ en relación con las funciones de la argumentación.

Las características textuales según el tipo discursivo predominante deberían ser tenidas en cuenta por los docentes tanto al seleccionar el material bibliográfico y al plantearse el trabajo en las aulas universitarias en el marco de cada asignatura. En este sentido, creemos que los niveles de análisis propuestos por Padilla, Douglas y López (2007; 2011) constituyen una herramienta didáctica interesante ya que permiten abordar los textos considerando los aspectos pragmáticos o contextuales, las diferentes formas de organización discursiva a nivel global y las marcas características del tipo discursivo a nivel local. Aunque un análisis detallado como el antes presentado excedería tal vez las posibilidades de trabajo en contextos áulicos, creemos que el reconocimiento de estos aspectos por parte de los estudiantes, así como de la clase textual, viabilizaría la construcción de la coherencia textual por lo que su enseñanza no debiera pasarse por alto.

\section{Conclusiones}

En el presente trabajo hemos analizado el tipo de textos que se utiliza en las nueve asignaturas del primer año de la carrera de psicología de una universidad estatal. Los hallazgos nos permiten apreciar la diversidad de clases textuales, de tipos discursivos y de formas de presentación y de organización del material bibliográfico seleccionado en estas materias de primer año. Hemos encontrado un predominio de textos académicos derivados de textos científicos y la presencia de textos predominantemente expositivos frente a los argumentativos. Además, este material bibliográfico no siempre cuenta con elementos que ayuden a contextuarlo y a veces los textos no se encuentran completos. En este sentido, surgen una serie de interrogantes: ¿cómo contextuar un texto cuando se encuentra desprovisto de referencias completas, datos de edición, índice, tapa, contratapa y solapas?, ¿cómo comprender el planteo de un autor cuando se trabaja con textos incompletos?, ¿los textos de predominio expositivo mediatizan a los de predominio argumentativo?, ¿qué tanto se alejan las prácticas de lectura del nivel secundario de las prácticas académicas del nivel universitario? 
Las características del material de lectura que los docentes proveen a los estudiantes -en cuanto a la falta de elementos para contextuar lo que se lee y a la incompletud del material- parece constituirse en una práctica institucionalizada y generalizada en varios espacios de cátedra. En este sentido, cabría indagar si en esta comunidad en particular, la enseñanza y el aprendizaje de la lectura se ven obstaculizados ya que la forma de preparación del material se aleja de lo indicado por los referentes teóricos citados. Asimismo, para los nóveles lectores el tipo discursivo de los textos puede constituirse en el mayor desafío, especialmente en el caso de los textos que argumentan. Creemos que si el propósito es que los estudiantes logren familiarizarse e incluirse como lectores académicos, los docentes debieran tener en cuenta esto y explicitar en las clases pautas para leer dichos textos. En este sentido, el trabajo en clases considerando los niveles de análisis del discurso expositivo y argumentativo de Padilla, Douglas y López (2007, 2011) puede constituir una herramienta para facilitar tanto los procesos de comprensión de lo leído como los de enseñanza de la lectura en el marco de la disciplina psicológica y en la comunidad de referencia. Este heurístico permitiría a los docentes y a los estudiantes tener en cuenta la interrelación entre los aspectos pragmáticos -casi no considerados por gran parte de los espacios de cátedra al preparar el material-, las características que adopta la organización del discurso en función del tipo discursivo predominante y las pistas lingüísticas locales propias de cada tipo discursivo en la construcción del sentido de lo que se lee.

Coincidimos con Carlino (2005) y con Araujo y Gomes Bezerra (2013) en señalar que aprender a leer y enseñar a leer diferentes clases textuales y tipos discursivos académicos constituye uno de los mayores desafíos para lograr incluir real y efectivamente a los estudiantes más y menos familiarizados con la cultura académica en las comunidades disciplinares. Creemos que un primer paso para concretar este anhelo de inclusión es la preparación cuidadosa del material de lectura que los docentes brindamos a los estudiantes, ya que éste vehiculiza los conocimientos disciplinares.

Por último, cabe señalar, que en estudios posteriores se prevé abordar algunos interrogantes que se desprenden de este estudio: ¿hay distancias entre los modos de lectura exigidos por los profesores en la universidad y los que los alumnos han construido en la secundaria?, ¿cómo inciden las perspectivas sobre la lectura en las prácticas de enseñanza y de aprendizaje?, ¿hay posibilidades de ocuparse de la lectura en las aulas universitarias?, ¿qué tipo de ayudas pedagógicas proporcionan los docentes y qué tipo de ayudas esperan recibir los alumnos?, ¿qué estrategias lectoras han desarrollado los estudiantes antes de ingresar a la universidad? 


\section{Referencias}

ARAUJO, Camila; GOMES BEZERRA, Benedito. Letramentos acadêmicos: leitura e escritura de gêneros acadêmicos no primeiro ano do curso de letras. Diálogos: Revista de Estudos Culturais e da Contemporaneidade, Garanhuns, v. 1, n. 9, p. 5-37, 2013.

ARNOUX, Elvira; DI STEFANO, Mariana; PEREIRA, María Cecilia. La lectura y la escritura en la universidad. Buenos Aires: Eudeba, 2002.

BARTON, David; HAMILTON, Mary; IVANIC, Roz. Situated literacies: reading and writing in context. London: Routledge, 2000.

BARTON, David; TUSTING, Karin. Beyond communities of practice: language, power and social contexts. New York: Cambridge University Press, 2005.

BAZERMAN, Charles. Shaping written knowledge. Madison: University of Wisconsin Press, 1988.

BIGI, Elisa, CHACÓN, Edixon; GARCÍA, Marisol. ¿Cuáles textos académicos leen los estudiantes universitarios en educación? Legenda, Mérida, v. 17, n., 17, p. 127-145, 2013.

BLOMMAERT, Jan et al. Academic literacies -what have we achieved and where to from here? Journal of Applied Linguistics, Oxford, v. 1, n. 4, p. 137-148, 2007.

CARLINO, Paula. Alfabetización académica: un cambio necesario, algunas alternativas posibles. Educere, Mérida, n. 20, p. 409-420, 2003b.

CARLINO, Paula. Escribir, leer y aprender en la universidad: una introducción a la alfabetización académica. Buenos Aires: Fondo de Cultura Económica, 2005.

CARLINO, Paula. Leer textos científicos y académicos en la educación superior: obstáculos y bienvenidas a una cultura nueva. In: CONGRESO INTERNACIONAL DE PROMOCIÓN DE LA LECTURA Y EL LIBRO, 7., 2003, Buenos Aires. Actas... Buenos Aires: Gobierno de la Ciudad de Buenos Aires, 2003a. p. 1-12.

CARLSON, Neal. Fisiología de la conducta. España: Addison Wesley, 2005.

CASSANY, Daniel. Literacidad crítica: leer y escribir la ideología. In: SIMPOSIO INTERNACIONAL DE LA SOCIEDAD ESPAÑOLA DE DIDÁCTICA DE LA LENGUA Y LA LITERATURA, 9., 2005, La Rioja. Actas... La Rioja: SEDELL/Universidad de la Rioja, 2005. p. 1-15.

CHIROLEU, Adriana. La inclusión en la Educación Superior como política pública: tres experiencias en América Latina. Revista Iberoamericana de Educación, México, v. 5, n. 48, p. 1-15, 2009b.

CHIROLEU, Adriana. Políticas públicas de inclusión en la educación superior: Ios casos de Argentina y Brasil. Pro-Posições, Campinas, v. 20, n. 2, p. 141-166, 2009a.

DE VEGA, Manuel. Introducción. In: DE VEGA, Manuel (Ed.). Introducción a la psicología cognitiva. Madrid: Alianza, 1984. p. 23-60.

ESTIENNE, Viviana; CARLINO, Paula. Leer en la universidad: enseñar y aprender una nueva cultura. Uni/pluiversidad, Antioquía, v. 4, n. 3, p. 1-13, 2004.

EZCURRA, Ana María. Igualdad en educación superior: un desafío mundial. Buenos Aires: Universidad Nacional de General Sarmiento: IEC/CONADU, 2011.

GIUDICE, Jacqueline. La adquisición del lenguaje disciplinar y académico en el inicio de los estudios universitarios: análisis de una experiencia educativa. In: CONGRESO NACIONAL CÁTEDRA UNESCO PARA EL MEJORAMIENTO DE LA CALIDAD Y EQUIDAD DE LA EDUCACIÓN EN AMÉRICA LATINA, CON BASE EN LA LECTURA Y LA ESCRITURA, 2014, Rosario. Actas... Rosario: Universidad Nacional de Rosario, 2014. p. 24-25.

GODOY, Marcelo; GIUDICE, Jacqueline. Problemática de los docentes en relación a las prácticas de lectura y escritura de sus alumnos universitarios y una modalidad de abordaje desde la gestión. In: CONGRESO NACIONAL CÁTEDRA UNESCO PARA EL MEJORAMIENTO DE LA CALIDAD Y EQUIDAD DE LA EDUCACIÓN EN AMÉRICA LATINA, CON BASE EN LA LECTURA Y LA 
ESCRITURA, 2014, Rosario. Actas... Rosario: Universidad Nacional de Rosario, 2014. p. 25.

HERNANDEZ SAMPIERI, Roberto; FERNÁNDEZ COLLADO, Carlos; BAPTISTA LUCIO, Pilar. Metodología de la investigación. México: McGraw and Hill: Interamericana, 2006.

KAUFMAN, Ana María. Escribir en la escuela: qué, cómo y para quién. Lectura y Vida, La Plata, n. 3, p. 2-18, 1994.

KAUFMAN, Ana María; RODRÍGUEZ, María Elena. La escuela y los textos: una clasificación de los textos que circulan en el entorno social sencilla y precia, articulada con una propuesta didáctica para que los niños lleguen a ser buenos lectores y escriban con corrección y autonomía. Buenos Aires: Santillana, 1993.

LEA, Mary. Communities of practice in higher education: useful heuristic or educational model? In: BARTON, David; TUSTING, Karin (Ed.). Beyond communities of practice. Cambridge: Cambridge University Press, 2005. p. 180-197.

LILLIS, Theresa; SCOTT, Mary. Defining academic literacies research: issues of epistemology, ideology and strategy. Journal of Applied Linguistics, Oxford, n. 4, p. 5-32, 2007.

MARIN, Marta. Alfabetización académica temprana. Lectura y Vida, La Plata, v. 27, n. 4, p. 30-39, 2006.

PADILLA, Constanza. Taller de comprensión y producción de textos. Tucumán: Facultad de Filosofía y Letras. Universidad Nacional de Tucumán, 2013. Ficha de clasificación de discursos. Material de uso interno.

PADILLA, Constanza; DOUGLAS, Silvina; LÓPEZ, Esther. Yo argumento: taller de prácticas de comprensión y producción de textos argumentativos. Córdoba: Comunicarte, 2011.

PADILLA, Constanza; DOUGLAS, Silvina; LÓPEZ, Esther. Yo expongo: taller de prácticas de comprensión y producción de textos expositivos. Córdoba: Comunicarte, 2007.

PEREZ ABRIL, Mauricio; RINCON BONILLA, Gloria. Para qué se lee y se escribe en la universidad. Bogotá: Pontificia Universidad Javeriana, 2013.

SCHUTZ, Alfred. El forastero: ensayo de psicología social. In: SCHUTZ, Alfred (Ed.). Estudios sobre teoría social. Buenos Aires: Amorrortu, 1974. p. 95-107.

Recibido en: 17.06.2015

Aprobado en: 16.02.2016

María Micaela Villalonga Penna es magíster en psicología cognitiva y educación por la Facultad Latinoamericana de Ciencias Sociales (FLACSO). Instituto de Investigaciones sobre el Lenguaje y la Cultura del Consejo Nacional de Investigaciones Científicas y Técnicas (CONICET).

Constanza Padilla es doctora en letras por la Universidad Nacional de Tucumán. Instituto de Investigaciones sobre el Lenguaje y la Cultura del Consejo Nacional de Investigaciones Científicas y Técnicas (CONICET). Facultad de Filosofía y Letras de la Universidad Nacional de Tucumán. 